\title{
Deformations and Displacements of the LHC Superconducting Dipoles Induced by Standard and Non-Standard Operational Modes
}

\author{
Marco La China, Juan Garcia Perez, Giuseppe Gubello, and Walter Scandale
}

\begin{abstract}
A full-scale and fully-instrumented working model of the LHC lattice cell has been tested at CERN between March and December 2002. Aside of the current, pressure and temperature sensors, controlled by an industrial supervision system, a novel device has been set to monitor magnet positions with respect to the surrounding cryostat. The series of operating modes to test cryogenics, current leads and quench recovery electronics offered the chance to investigate potentially harmful deformations of the superconducting structure. In this paper we present a survey of displacements and deformations experienced by the LHC cell magnets during thermal cycles, current ramps and resistive transitions. Although the system complexity prevented from complete modeling, a preliminary phenomena explanation is given.
\end{abstract}

Index Terms-Alignment, cold test, dipole deformations.

\section{INTRODUCTION}

$\mathbf{T}$ HE Large Hadron Collider will accelerate two counter rotating protons beams along a $27 \mathrm{~km}$ long circular trajectory to provide particle-particle interaction at $14 \mathrm{TeV}$ collision energy. To bend such high-energy beams, an $8.3 \mathrm{~T}$ dipolar field will be generated by 1232 superconducting magnets placed along the theoretical particle trajectory. The $15 \mathrm{~m}$-long dipoles have a curved shape, designed to closely follow the circular beam trajectory. In addition, multipolar correctors are welded at the dipole ends to locally compensate the higher field shape harmonics, unavoidable in superconducting magnets. In spite of the deformations induced by thermal contraction and Lorentz forces, the geometrical constraints should guarantee that the multipolar corrector positions are close to the beam orbit within a strict tolerance $(0.3 \mathrm{~mm}$ in average and 0.5 in $\mathrm{rms})$, that the mechanical clearance is always larger than 6 times the beam radius and that the interconnecting bellows (withstanding misalignment up to $4 \mathrm{~mm}$ ) have tolerable radial offset.

To monitor the deformation of a mechanical structure generating an $8.3 \mathrm{~T}$ field at $1.9 \mathrm{~K}$, insulated by thermal shields and housed in a cryostat, a specific measuring system has been developed at CERN in collaboration with external partners [3]. Magnet displacements and deformations were measured in operative phases during the test of the first LHC full-cell prototype called String2, schematically shown in Fig. 1. This full-scale working model, has undergone typical and atypical operating

Manuscript received January 20, 2003.

The authors are with CERN (European Center for Nuclear Research) $\mathrm{CH}$ 1211 Geneve, Switzerland (e-mail: marco.la.china@cern.ch).

Digital Object Identifier 10.1109/TASC.2004.829049

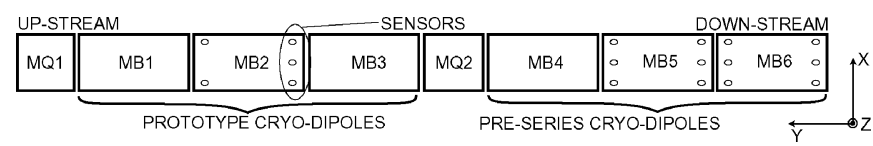

Fig. 1. String2 magnets layout (LHC standard cell).

modes during which the cryogenic apparatus, the magnet electrical circuits and the quench recovery systems have been tested to check their performance and to validate the design choices. In this framework, through the acquisition of magnet extremity displacements in vertical and horizontal directions, it has been possible to identify the operating phases that could harmfully affect the magnet short and long-term mechanical stability. The network of sensors mounted on the string magnets is outlined in the next section whereas third section presents the effect of the energization on magnet position and shape. Quench effects are described in the fourth section and thermal related phenomena are discussed in the fifth. A summary of the detected phenomena is given in the sixth and final section.

\section{StRing2 MONitoring System}

In the choice of the monitoring system, the extreme environmental conditions represented by the vacuum and the $300 \mathrm{~K}$ thermal gradient between the cold-mass and the surrounding cryostat did not suggest common deformation sensors like strain gages or inductive comparators. In fact both of these would have acted as thermal bridge between cold-mass and cryostat and consequently suffered from deformations induced by very high thermal gradients. Moreover the magnet extremity six degrees of freedom would have made hard to distinguish a deformation along one direction from tilt and from deformations in other directions. These constraints pushed us toward a tailor made solution developed in collaboration with the EPFL (Ecole Polytechnique Federale de Lausanne) and SMARTEC S.A. [3]. In fact, the original idea to overcome all the previous issues by mean of an optical device was eventually realized through the modification of a commercial measuring device used to monitor deformations in civil structures and already in production by SMARTEC S.A..

The LHC cell represented by String2 is made up of two half cells each one including one quadrupole magnet (MQ) and three dipole magnets (MB) with their correctors. To reproduce the regular part of an LHC arc, String2 was assembled by aligning the magnets along a theoretical curved path as in the LHC tunnel. It is important to notice that in String2 the two half cells are not identical since the older one is made up of 

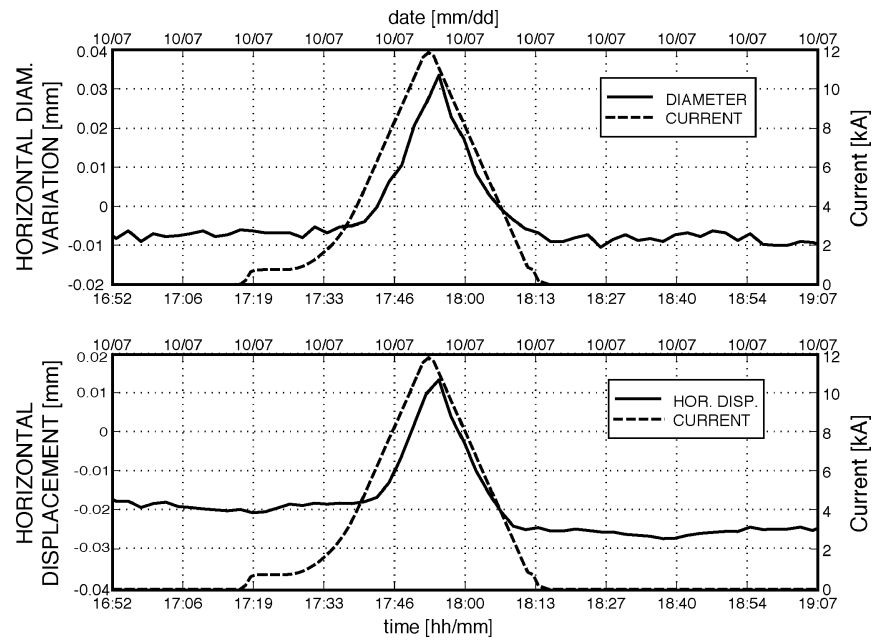

Fig. 2. Energization effect on horizontal displacement and outer cold-mass diameter variation of prototype magnet MB2.

prototype dipoles that already formed the String1 half-cell, whereas the new one is made of pre-series dipoles of final design and construction. To reveal different behavior between prototype and pre-series magnets, the network of optical sensors was installed on both kinds of magnets. The magnet layout and the sensor locations are visible in Fig. 1 along with the reference system.

To monitor vertical and horizontal position of the ends (that is along $\mathrm{Z}$ and $\mathrm{X}$ axis of Fig. 1 reference system) as well as crosssection contraction, three sensors are needed for each magnet extremity. Three sensors were installed in both ends of the preseries MB5 and MB6 and in the down stream end of the prototype MB2 whereas in its up stream end only two sensors were mounted so that no vertical displacement could be detected in that extremity.

\section{STRING POWERING}

Before reaching the nominal energy, particles have to travel several million turns at increasing momentum so that, to keep them in the same circular trajectory, an increasing dipolar field is required. In this 20 min-long transient phase, the increasing electro-magnetic forces can modify the stress distribution inside the coils and may provoke a slight change of the cold-mass curvature and a consequent sizable misalignment of the extremities in radial and vertical directions. Both phenomena have been detected during the energization of the main magnet circuits performed in the cold test facility.

The amount of collected data, once manipulated to make them homogeneous, revealed a reproducible behavior of magnet ends in terms of displacements and cross-section deformation. The typical effect of current ramping on the coldmass cross-section is displayed in the top graph of Fig. 2 where the exciting current and the horizontal diameter of a pre-series magnet are plotted versus the time. The tight correlation between diameter variation and current level is evident in spite of the horizontal shift of few minutes due to different settings of measuring system clocks. Diameter variation is due to the electro-magnetic forces acting on the coils and pushing them along the top-bottom symmetry axis of the cross-section in the outward
TABLE I

ENERGIZATION-INDUCED DISPLACEMENTS

\begin{tabular}{ccccccc}
\hline \hline & $\mathrm{I}_{\max }[\mathrm{A}]:$ & 5000 & 8000 & 10800 & \multicolumn{2}{c}{11850} \\
& $n$ of meas.: & 4 & 1 & 2 & \multicolumn{2}{c}{14} \\
\hline magnet & def. $[\mathrm{mm}]$ & & & & mean & $r m s$ \\
\hline \multirow{2}{*}{ MB2 } & $\Delta \mathrm{d}_{\max }$ & $<0.01$ & 0.02 & 0.02 & 0.04 & 0.002 \\
& $\Delta \mathrm{X}_{\max }$ & $<0.01$ & 0.01 & 0.03 & 0.04 & 0.002 \\
& $\Delta \mathrm{d}_{\max }$ & - & 0.02 & 0.03 & 0.03 & 0.004 \\
\multirow{3}{*}{ MB5 } & $\Delta \mathrm{X}_{\max }$ & - & $<0.01$ & 0.03 & 0.06 & 0.03 \\
& $\Delta \mathrm{Z}_{\max }$ & -0.01 & 0.08 & -0.14 & -0.09 & 0.04 \\
\hline \hline
\end{tabular}

direction. By assuming a linear elastic behavior of the cold-mass the current-deformation relation that comes from the Lorentz equation is parabolic and precisely fits the experimental data. A clear current-driven behavior is also visible in the bottom part of Fig. 2 where the horizontal displacement of the pre-series dipole end is plotted along with the current ramp versus the time. In this case the relation cannot be quickly resolved in a quantitative way because of the three-dimensional nature of the phenomenon. However it can be interpreted as a straightening of the cold-mass in the horizontal plane due to the coil thrust that counteracts the elastic forces that keep the cold-mass bent. What must be pointed out first is the magnitude of the observed phenomena which is rather small with respect to the critical values that could affect the machine performance. Maximum displacements in fact, generally far below $0.1 \mathrm{~mm}$ in horizontal direction and around it in vertical direction, cannot result by themselves in a harmful misalignment of the multipolar correctors (that is $>0.3 \mathrm{~mm}$ ). Second point to be noted is the fully elastic feature of the displacements and deformations provoked by current-related phenomena. It is indeed clear, in both the figures, the absence of any significant residual effect on the diameter size as well as on the displacement once that the current is brought down to the original value. In Table I we summarize maximum displacements and deformations experienced by two string magnets for different current ramps. Data are about the down stream extremity of a pre-series and of a prototype magnet for which the vertical displacement is not available. From those data (averaged over a large number of measurements), we see that diameter variation $\Delta \mathrm{d}_{\max }$ and horizontal displacement $\Delta \mathrm{X}_{\max }$ seem to follow the same simple trend in both the magnets whereas the vertical displacement $\Delta \mathrm{Z}_{\max }$ shows a slightly larger size.

\section{STRING QUENCHES}

Quenches performed in the string have been provoked at different current intensities, on different magnets and with different current decay time rates. In spite of the different quench features, the analysis showed up quite well reproducible behavior for the diameter and for the horizontal displacements. Quenches are provoked by resistive heaters placed in specific locations of the coil and activated at pre-defined current values. It can be noticed that in the first part, that is the current ramping, there is no difference with respect to the energization phase. This is clearly visible in the top graph of Fig. 3 where the diameter variation is plotted, along with the current, 

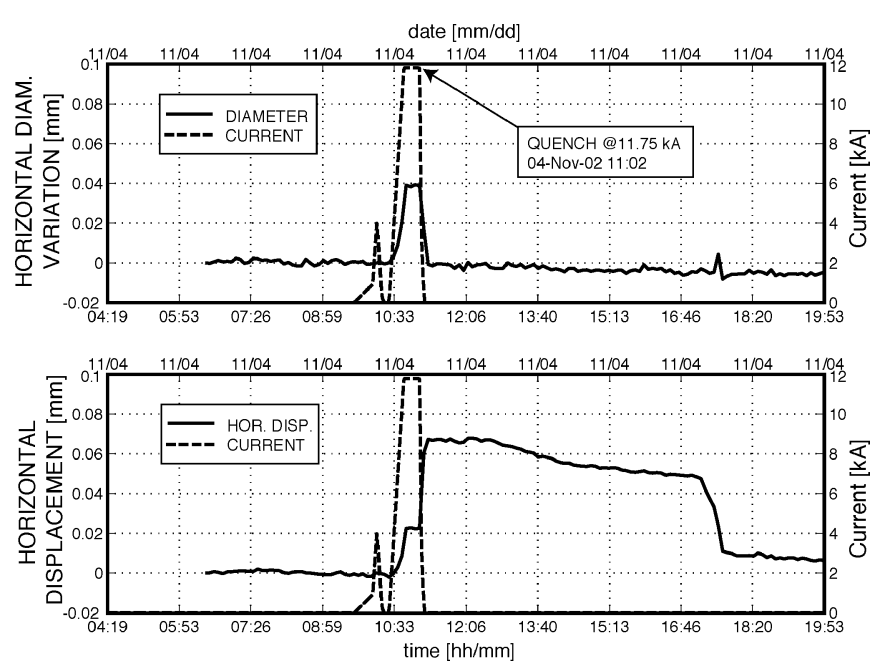

Fig. 3. Horizontal displacement and diameter variation induced by quench at $11.75 \mathrm{kA}$.

versus the time. After the preliminary part, in which the current raises with a fixed time-rate value, the current is kept constant until the quench is provoked and then it is cut off by the protection system. We notice that the diameter closely follows the current profile so that after the current cut no residual deformation is present in the magnet cross-section. The magnet extremity horizontal displacement for the same quench test is visible in the bottom graph of Fig. 3. The behavior is the same for the current ramp until the quench occurs. After the quench and the consequent current cut, the magnet end experiences a sizable displacement in the horizontal outward direction of about $0.7 \mathrm{~mm}$, recovered only much later. Since that happens after the quench it cannot be obviously related to electro-magnetic forces and a plausible explanation can be found in the thermal-driven phenomena. One of the main outcomes of a resistive transition is, indeed, the release of a large amount of magnetic energy dissipated as heat in the coil. Moreover, at very low temperatures the specific heat and the thermal conductivity of metals are both low so that a little amount of energy can cause a relevant and localized increase of temperature in the structure. This can be seen in Fig. 4 where the magnet temperature, measured in proximity of the quench spot, and the end displacement are plotted versus the time. As soon as the quench takes place the current drops and the temperature rises very quickly and so does the horizontal displacement. The amount of stored energy needs a long time to be extracted by the helium flow and, as visible in the plot, more or less the same time is needed by the magnet to regain its original position. The relation between thermal and structural phenomena isthus evident even if a quantitative model is not straightforward. The recovery time, reported in Table II along with a summary of the collected data, is generally around 3.8 hours with an rms spread of about 0.8 hours. The maximum displacement, usually reached just after the energy release by the coil resistive part, is not very large in the horizontal direction $\left(\Delta \mathrm{X}_{\max }\right)$ whereas is almost critical in the vertical direction $\left(\Delta \mathrm{Z}_{\max }\right)$. In spite of the few (5) available data the reproducibility is quite good and the average value is set close to the critical $0.3 \mathrm{~mm}$. In operative condition,

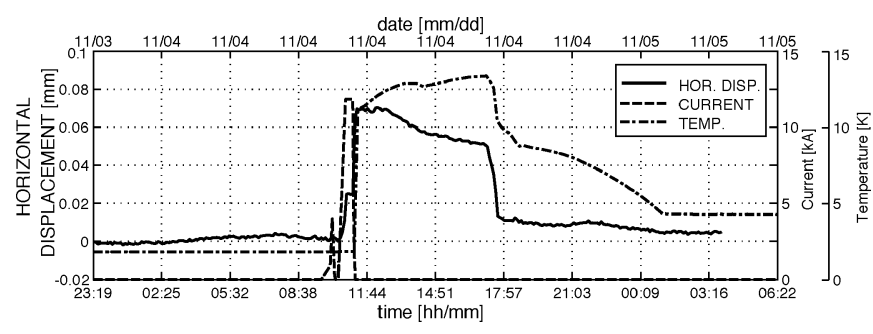

Fig. 4. Horizontal shift and temperature raise provoked by quench at $11.75 \mathrm{kA}$.

TABLE II

DISPLACEMENTS INDUCED BY QUENCHES

\begin{tabular}{cccc}
\hline \hline & average & $r m s$ & n of meas. \\
\hline$\Delta \mathrm{X}_{\max }$ & $0.07 \mathrm{~mm}$ & $0.02 \mathrm{~mm}$ & \multirow{2}{*}{23} \\
Recovery Time & 3.8 hours & 0.8 hours & \\
$\Delta \mathrm{Z}_{\max }$ & $-0.24 \mathrm{~mm}$ & $0.03 \mathrm{~mm}$ & 5 \\
Recovery Time & 3.6 hours & 0.7 hours & 5 \\
\hline \hline
\end{tabular}

anyhow, such a sizable misalignment is reached when the beam has already been dumped because of the quench and the recovery toward the original position, accomplished after the recovery time, ensures that there is no real danger for beam stability related to this effect.

\section{STRING COOLDOWN AND WARMUP}

The effects of thermal gradients along the magnets can be made clearly visible in the cooling phase, needed to bring the magnet structure down to the operational temperature of $1.9 \mathrm{~K}$. In fact the cooldown phase, is performed by flowing helium through the magnets. Cold gas is injected from one end (upstream) at decreasing temperature and extracted from the opposite (down-stream) [4]. The cooldown rate triggers the longitudinal thermal gradient with a consequent structural deformation of the dipole. Both fast or slow cooldowns were already proved to provoke shift of magnet end positions in previous tests performed either on prototype and on pre-series magnets as reported in [5]. In those occasions nonnegligible displacements affecting the magnet also after the transient were detected during fast cooldowns in prototype magnets. The most unfavorable event indeed was a displacement of $2 \mathrm{~mm}$ peak in horizontal direction experienced by a prototype magnet during a fast thermal transient.

In the run of the String2 the cooldown was of slow rate in order to keep the thermal gradient along a single magnet below $80 \mathrm{~K}$ and to limit potentially harmful thermo-mechanical stresses. The measured displacements in vertical and horizontal direction are reported, along with the diameter contraction, in Table III. In spite of the lack of data due to temporary sensor failures, it can be noticed a diameter contraction $\Delta$ d close to the one expected $(1.2 \mathrm{~mm})$ and the presence of nonzero displacements in both directions once terminated the transient phase. In vertical direction $\left(\Delta \mathrm{Z}_{\text {residual }}\right)$ they are induced by support post and cold mass shrinking $(0.3$ and $0.6 \mathrm{~mm}$, respectively) and close to what expected, whereas in horizontal direction $\left(\Delta \mathrm{X}_{\text {residual }}\right)$ they can reach values and are not fully understood yet. To check if the misalignments provoked by cooldown are still present after the string warming up to 
TABLE III

DISPLACEMENTS INDUCED BY SLOW COOLDOWN

\begin{tabular}{|c|c|c|c|c|}
\hline end & $\operatorname{def}[\mathrm{mm}]$ & MB2 & MB5 & MB6 \\
\hline \multirow{5}{*}{ 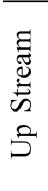 } & $\Delta \mathrm{d}$ & & -1.14 & \\
\hline & $\Delta \mathrm{X}_{\max }$ & & -0.30 & \\
\hline & $\Delta \mathrm{X}_{\text {residual }}$ & & -0.20 & \\
\hline & $\Delta Z_{\max }$ & & -1.37 & \\
\hline & $\Delta Z_{\text {residual }}$ & & -0.92 & \\
\hline \multirow{5}{*}{ 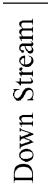 } & $\Delta \mathrm{d}$ & & & -1.00 \\
\hline & $\Delta \mathrm{X}_{\max }$ & & & 0.10 \\
\hline & $\Delta \mathrm{X}_{\text {residual }}$ & 0.40 & 0.20 & 0.02 \\
\hline & $\Delta Z_{\max }$ & & -1.20 & \\
\hline & $\Delta Z_{\text {residual }}$ & & -1.10 & \\
\hline
\end{tabular}

TABLE IV

ResiduAl DisPlacements AFTER STRING SHUT-DOWN

\begin{tabular}{ccccc}
\hline \hline & \multicolumn{2}{c}{ Up Stream } & \multicolumn{2}{c}{ Down Stream } \\
& $\Delta \mathrm{X}[\mathrm{mm}]$ & $\Delta \mathrm{Z}[\mathrm{mm}]$ & $\Delta \mathrm{X}[\mathrm{mm}]$ & $\Delta \mathrm{Z}[\mathrm{mm}]$ \\
\hline MB2 & 0.02 & - & -0.04 & -0.07 \\
MB5 & 0.20 & -0.10 & -0.03 & -0.07 \\
MB6 & -0.06 & - & - & - \\
\hline \hline
\end{tabular}

the room temperature one can look at Table IV in which are reported the measurement taken right after the string shut down. It can be noticed that the measured displacements are usually around few hundredths of millimeter and always smaller than the imposed tolerance range.

\section{CONCLUSION}

To ensure the respect of the imposed tolerances on alignment and shape of the main LHC dipole, three coldmasses of the LHC cell prototype String2 were equipped with specifically developed optical sensors. Coldmass end positions were thus monitored with respect to the cryostat during the whole series of test performed on String2 to reproduce standard working con- ditions as well as risky occurrences as, for example, propagation of resistive transitions. During energization the maximum horizontal displacements did not exceed $0.05 \mathrm{~mm}$ whereas in vertical direction they reached $-0.1 \mathrm{~mm}$. Effects of quenches led to larger displacements due to the release of thermal energy in the conductors. Maximum misalignments of the ends in vertical and horizontal direction, around $0.1 \mathrm{~mm}$ and $0.2 \mathrm{~mm}$ respectively, were considered safe since they will occur when the beam will be already dumped. Such displacements are usually recovered as soon as a uniform temperature along the magnet is restored as generally happens after some hours. More critical effects are related to the deformations induced by thermal transients. During nominal cooldown rate they can reach values of some tenths of millimeter (at the maximum of the temporary longitudinal thermal gradient) and can sporadically lead to small residual misalignments. It must be nevertheless pointed out that the worst cases were generally represented by displacement never exceeding $0.3 \mathrm{~mm}$, hence tolerable for beam dynamics.

A last note is that small residual deformations and displacements induced by cooldown can persist even after the opposite thermal transient (warm up).

\section{REFERENCES}

[1] W. Scandale, M. Bajko, and F. Savary, "Geometry and alignment requirements for the LHC main dipole," in 7th European Particle Accelerator Conference, 2000, pp. 2160-2163.

[2] R. Bartolini and W. Scandale, Multipole feed-down due to dipole misalignments, in CERN LHC MMS Internal note 97-12, 1997.

[3] D. Inaudi et al., "Development of a displacement sensor for the CERN-LHC superconducting cryodipoles," Meas. Sci. Technol., vol. 12, pp. 887-896, 2001.

[4] P. Lebrun, G. Riddone, L. Tavian, and U. Wagner, "Cooldown and warmup studies for the Large Hadron Collider," in Proc. of 17th International Cryogenic Engineering Conference, Bournemouth, UK, Jul. 14-17, 1998, pp. 813-816.

[5] W. Scandale, "Optical in-situ measurement of relative deformations of the LHC main dipole cold masses," IEEE Trans. Appl. Superconduct., vol. 12, no. 1, pp. 1736-1740, March 2002. 